\title{
TRATAMENTO CIRÚRGICO DE RECIDIVAS DE HÉRNIAS DISCAIS LOMBARES: QUE RESULTADOS?
}

\author{
SURGICAL TREATMENT IN RECURRENT LUMBAR DISC HERNIATION: WHAT RESULTS? \\ TRATAMIENTO QUIRÚRGICO DE RECIDIVAS DE HERNIAS LUMBARES DE DISCO: CUÁLES \\ SON LOS RESULTADOS?
}

Pedro Alberto Pinto da Silva', Paulo Miguel Silva Pereira ${ }^{1}$, Patrícia Maria Polónia Pinto', Rui Manuel Cardoso Vaz ${ }^{1}$

\begin{abstract}
RESUMO
Objetivos: No tratamento da recidiva de hérnia discal lombar, em particular no âmbito cirúrgico, vários aspectos se mantêm controversos. O presente trabalho pretende contribuir para definir características pré-operatórias que influenciem os resultados subjetivo e objetivo da cirurgia. Métodos: Foram selecionados doentes submetidos à intervenção cirúrgica por hérnia discal lombar recidivada em um período de dez anos; foram revistos os processos clínicos e reavaliados os pacientes, de forma subjetiva (grau de satisfação, Pain Visual Analogue Scale, Score de Stanford) e objetiva (Índice de Incapacidade de Oswestry, Questionário de Zurich). Foi realizada uma análise estatística desses dados. Resultados: O número total foi de 55 doentes, com predomínio do sexo masculino. A taxa de complicações foi de 7,3\%. Onze doentes (20\%) necessitaram de terceira intervenção cirúrgica. A grande maioria (91,5\%) dos doentes afirma-se satisfeita com o tratamento cirúrgico. Houve uma variação média favorável pelo Índice de Oswestry (-46,27\%), confirmada pelas restantes escalas; $81,6 \%$ dos pacientes ativos retomaram a atividade laboral prévia. Foram encontrados preditores significativos do resultado funcional, e a necessidade de terceira cirurgia para o retorno à atividade laboral. Conclusões: O tratamento cirúrgico da recidiva de hérnia discal lombar permite resultados favoráveis no controle sintomático e funcional, em todos os testes aplicados. Algumas variáveis pré-operatórias podem ajudar a prever os pacientes menos susceptíveis à melhoria.
\end{abstract}

Descritores: Recidiva; Deslocamento do disco intervertebral/diagnóstico; Deslocamento do disco intervertebral/cirurgia; Ciática; Satisfação do paciente.

\begin{abstract}
Objectives: Concerning treatment for recurrent lumbar disc herniation, especially in surgery, several aspects remain controversial. This work aims to define preoperative characteristics that influence the subjective and objective results of surgery. Methods: We selected patients undergoing surgery for recurrent lumbar disc herniation over a period of ten years; we reviewed the medical records and reassessed the patients subjectively (degree of satisfaction, Pain Visual Analogue Scale, Stanford Score) and objectively (Oswestry Disability Index, Zurich Questionnaire). A statistical analysis of these data was carried out. Results: The total number was 55 patients, predominantly male. The complication rate was $7.3 \%$. Eleven patients (20\%) needed a third surgery. A large majority (91.5\%) of patients said they were satisfied with the surgical treatment. There was a favorable average variation of the Oswestry Index (-46.27\%), confirmed by other scales; $81.6 \%$ of active patients resumed previous work activity. Significant predictors of functional outcome were found and also the need of a third surgical procedure for the return to work activity. Conclusions: Surgical treatment for recurrent lumbar disc herniation allows a favorable outcome in symptomatic and functional terms in all tests. Some preoperative variables can help predict patients less susceptible to improvement.
\end{abstract}

Keywords: Recurrence; Intervertebral disk displacement/diagnosis; Intervertebral disk displacement/surgery; Sciatic; Patient satisfaction.

\section{RESUMEN}

Objetivos: En el tratamiento de la hernia de disco lumbar recurrente, especialmente en relación con la cirugía, algunos aspectos siguen generando controversias. Este trabajo tiene por objetivo definir las características preoperatorias que influyen en los resultados subjetivos y objetivos de la cirugía. Métodos: Seleccionamos los pacientes sometidos a cirugía de hernia de disco lumbar recurrente durante un período de diez años. Fueran revisados los registros médicos y se reevaluó a los pacientes subjetiva (grado de satisfacción, escala visual analógica del dolor, Score de Stanford) y objetivamente (Índice de discapacidad de Oswestry, Cuestionario de Zurich). Se realizó un análisis estadístico de estos datos. Resultados: El número total fue 55 pacientes, predominantemente de sexo masculino. La tasa de complicaciones fue 7,3\%. 11 pacientes (20\%) necesitaron una tercera cirugía. Una gran mayoría (91,5\%) de los pacientes mostró su satisfacción con el tratamiento quirúrgico. Se verificó una variación promedio favorable del Índice de Oswestry (-46,27\%), confirmada por las otras escalas; el $81,6 \%$ de los pacientes activos reanudó la actividad laboral previa. Fueran encontrados factores predictivos significativos de los resultados funcionales, necesidad de una tercera intervención quirúrgica y para el regreso al trabajo. Conclusiones: El tratamiento quirúrgico de la hernia discal lumbar recurrente permite un resultado favorable en el control sintomático y funcional en todas las pruebas. Algunas variables preoperatorias pueden ayudar a predecir los pacientes menos susceptibles de mejora.

Descriptores: Recurrencia; Desplazamiento del disco intervertebral/diagnóstico; Desplazamiento del disco intervertebral/cirugía; Ciática; Satisfacción del paciente. 


\section{INTRODUÇÃO}

As hérnias discais lombares recidivadas constituem uma entidade nosológica frequente mas complexa, com que todo o cirurgião raquidiano tem de lidar. Além de implicações importantes na capacidade funcional e na qualidade de vida do paciente, o contexto e o modo como se apresentam influem de forma importante na relação médico-doente e na avaliação que este faz do seu processo terapêutico.

Apesar da sua incidência e de extensa literatura publicada sobre o tema, persistem controvérsias quanto à sua epidemiologia, opções terapêuticas e seus resultados.

Os critérios para reintervenção cirúrgica e a técnica a utilizar são menos consensuais que no caso de uma primeira intervenção e os resultados da cirurgia são igualmente menos homogéneos.

Importa, assim, identificar características inerentes ao paciente ou ao processo patológico que possam influenciar o sucesso da terapêutica cirúrgica, o resultado funcional e o grau de satisfação do doente com o tratamento.

\section{MÉTODOS}

Foram seleccionados os doentes submetidos a intervenção cirúrgica por hérnia discal lombar recidivada por cirurgiões do Serviço de Neurocirurgia do Hospital de S. João entre os anos de 1999 e 2008, independentemente do cirurgião e instituição envolvidos na primeira cirurgia.

Foi definida como recidiva de hérnia lombar a recorrência clínica e imagiológica do processo patológico após um primeiro tratamento cirúrgico com resultado favorável, no mesmo nível intervertebral lombar e independentemente da lateralidade das queixas.

Foram excluídos pacientes submetidos a segundo procedimento cirúrgico com artrodese ou instrumentação.

Foram revistos os processos clínicos e reavaliados os pacientes, sendo recolhidos dados biográficos, biométricos, clínicos e imagiológicos; foi registada também informação sobre os procedimentos efectuados. Procedeu-se à avaliação subjectiva dos resultados do tratamento, intensidade da dor lombar e no membro inferior pela Visual Analogue Scale ${ }^{1}$ (VAS), avaliação objectiva pré e pós-operatória pelo Índice de Incapacidade de Oswestry ${ }^{2}$ (ODI) e Questionário de Zurich $^{3}$, e à determinação do Score de Stanford ${ }^{4}$ e classificação dos resultados da intervenção cirúrgica pelos Critérios de Odom ${ }^{5}$.

No trabalho estatístico, utilizaram-se os testes $t$-Student ou Mann-Whitney na análise de associação entre uma variável categórica com duas categorias e uma variável numérica, respectivamente na presença ou na ausência de desvio relevante da normalidade na distribuição dentro dos grupos. No estudo da associação entre 2 variáveis numéricas foi usada a análise de correlação de Spearman. O teste do Qui-Quadrado foi aplicado para a associação entre duas variáveis categóricas. Foram aceites como significativos os valores de $p$ inferiores a 0,05 . Os dados foram processados utilizando a aplicação SPSS Statistics 17.0 (SPSS Inc.), trabalhando em Windows XP (Microsoft).

\section{RESULTADOS}

Com os critérios definidos previamente foram encontrados 55 pacientes (35 do sexo masculino e 20 do sexo feminino) submetidos a intervenção cirúrgica por hérnia discal lombar recidivada (Tabela 1). A média de idades à data do procedimento foi de 47,3 anos (entre 27 e 77).

O nível intervencionado foi L5-S1 em 29 casos (52,7\%), L4-L5 em 22 casos (40\%) e L3-L4 em 4 casos (7,3\%). Todos os pacientes foram submetidos a revisão da microdiscectomia e dissecção
Tabela 1. Características gerais da população.

\begin{tabular}{|c|c|c|c|}
\hline \multicolumn{2}{|c|}{ Características populacionais } & $\mathbf{N}$ & $\%$ \\
\hline \multirow{2}{*}{ Sexo } & Masculino & 35 & 63,6 \\
\hline & Feminino & 20 & 36,4 \\
\hline \multirow{6}{*}{ Escalão Etário } & $\leq 29$ & 2 & 3,6 \\
\hline & 30 a 39 & 12 & 21,8 \\
\hline & 40 a 49 & 23 & 41,8 \\
\hline & 50 a 59 & 8 & 14,5 \\
\hline & 60 a 69 & 6 & 10,9 \\
\hline & $\geq 70$ & 4 & 7,3 \\
\hline \multirow{4}{*}{$\begin{array}{c}\text { Nível } \\
\text { Intervencionado }\end{array}$} & L3-L4 & 4 & 7,3 \\
\hline & L4-L5 & 22 & 40 \\
\hline & L5-S1 & 29 & 52,7 \\
\hline & Total & 55 & \\
\hline
\end{tabular}

peri-radicular com sequestrectomia e descompressão óssea quando indicado.

O tempo médio entre procedimentos cirúrgicos foi de 58 meses (entre 2 e 148). A reintervenção cirúrgica foi mais frequente no primeiro ano após a primeira cirurgia $(n=15,27,2 \%)$, mantendo-se depois a incidência anual relativamente estável no primeiro quinquénio, decrescendo até ao fim da primeira década e tornando-se depois esporádica (Figura 1).

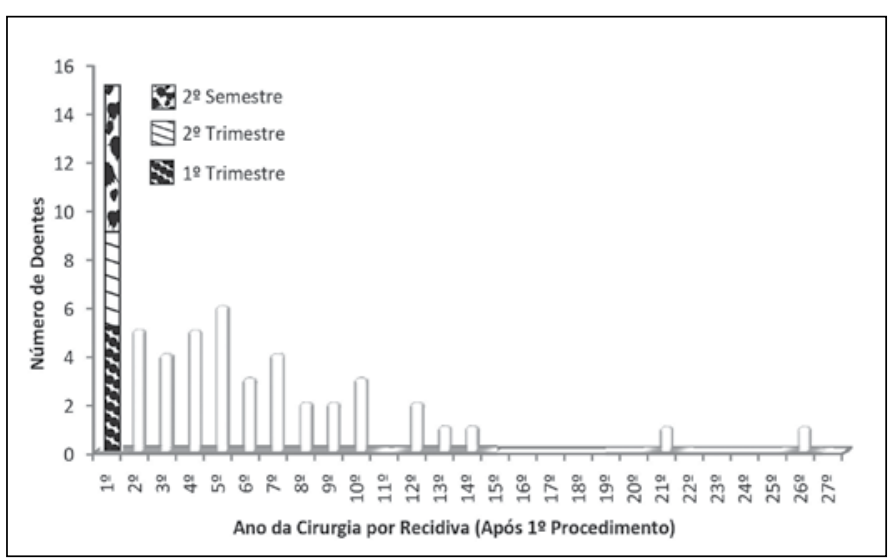

Figura 1. Temporalidade da cirurgia por recidiva.

O tempo médio livre de sintomas após a primeira cirurgia foi de 43,8 meses, e o período médio entre recorrência clínica e a execução da segunda intervenção cirúrgica foi de 14,2 meses.

O tempo médio de baixa laboral pré-operatório foi de 3,35 meses (entre 0 e 59). Todos os doentes apresentavam dor no membro inferior antes de primeira e segunda intervenções. Aquando da cirurgia por recidiva de hérnia discal, o sintoma predominante era a dor do membro inferior em $88,7 \%$ dos doentes, a lombalgia em $7,5 \%$ dos doentes, e nos restantes nenhuma das queixas predominava. Entre cirurgias verificou-se uma correspondência significativa em termos de frequência de achados clínicos (Figura 2).

A taxa de complicações cirúrgicas foi de 7,3\% $(n=4)$. Em dois casos ocorreu laceração dural, corrigida intra-operatoriamente e sem complicações subsequentes. Um paciente apresentou um novo défice sensitivo no pós-operatório; num outro doente ocorreu 


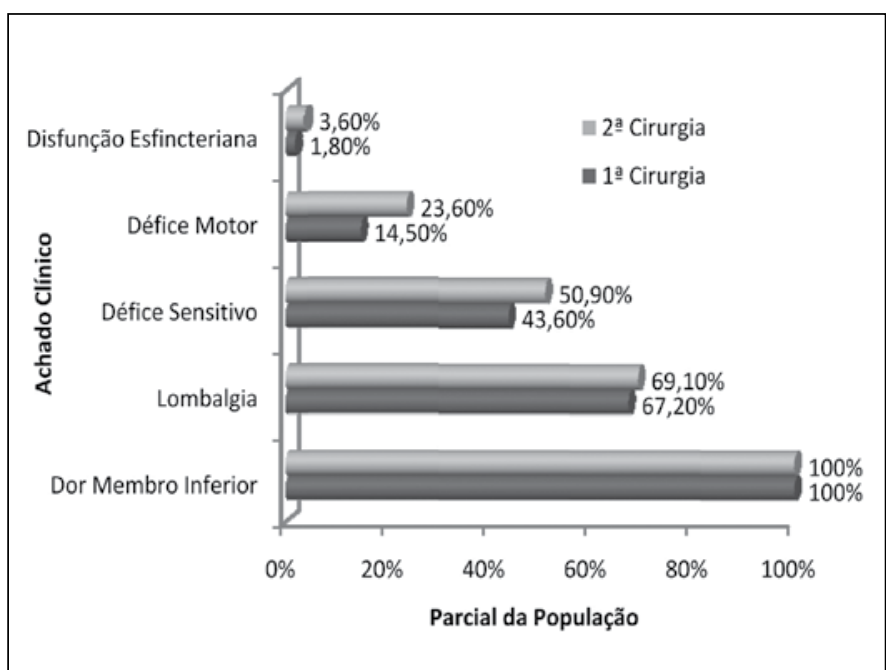

Figura 2. Achados clínicos pré-operatórios.

um episódio auto-limitado de retenção urinária. Não houve mortalidade per-operatória.

Uma terceira intervenção cirúrgica foi necessária em 11 doentes (20\%): 7 destes foram submetidos a re-abordagem e discectomia simples (um dos quais necessitou posteriormente de uma artrodese); os restantes 4 foram submetidos a artrodese lombar. O tempo médio entre segundo e terceiro procedimentos cirúrgicos foi de 26 meses.

O tempo médio de follow-up após cirurgia de recidiva foi de 38,7 meses.

Uma larga maioria $(91,5 \%)$ dos doentes afirma-se satisfeita com o tratamento cirúrgico e repeti-lo-ia se pudesse voltar atrás, conhecendo antecipadamente o resultado. O grau "Satisfação" do Score de Stanford médio foi de 8,26 em 10. A pontuação média da escala de Satisfação pelo Questionário de Zurich foi de 1,45 (numa escala de 1 a 4 , sendo 1 o melhor valor possível).

O VAS lombar médio à data de avaliação foi de 3,34 (numa escala de 0 a 10); o VAS para membro inferior foi de 2,74.

Dos pacientes activos e empregados, $81,6 \%$ retomaram a actividade laboral prévia. Em média, o tempo decorrido entre o procedimento e o retorno laboral foi de 3,6 meses.

Aproximadamente metade dos pacientes não faz uso, à data de reavaliação, de qualquer tipo de analgésico; cerca de $27 \%$ fá-lo de forma esporádica (menos que uma vez por mês); em $8 \%$ dos casos, no entanto, verifica-se uma necessidade diária de terapia médica antálgica. O grau "Uso de Medicação" do Score de Stanford médio foi de 8,46 em 10 (escala de 0 a 10, sendo 10 o melhor valor possível).

O Índice de Incapacidade de Oswestry médio pré-operatório era de $63,03 \%$. O valor pós-operatório encontrado foi de $16,76 \%$, correspondendo a uma variação média de -46,27\% (Figura 3A).

$\mathrm{Na}$ avaliação do resultado da cirurgia pelo Score de Stanford (Figura 3B), o valor médio total foi de 7,69/10; pontuações mais altas foram obtidas para os parâmetros "Medicação" (média de 8,46/10) e "Satisfação" (média de 8,26/10), seguidas dos parâmetros "Dor" e "Restrição às Actividades" (médias de 7/10).

A avaliação de resultados pelo Questionário de Zurich foi também favorável, com uma variação média de -1,29/5 na escala de Gravidade de Sintomas e -1,61/4 na escala de Função Física (Figura 3C).

Aplicando os Critérios de Odom, aproximadamente $80 \%$ dos resultados foram classificados como Excelente ou Bom, sendo que em quase 10\% dos casos o resultado foi Fraco (Figura 3D).

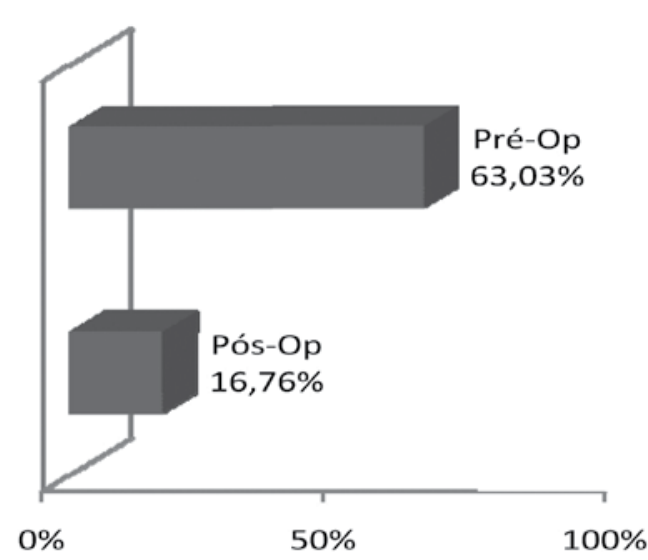

Indíce de Oswestry Médio

Figura 3A. Avaliação dos resultados.

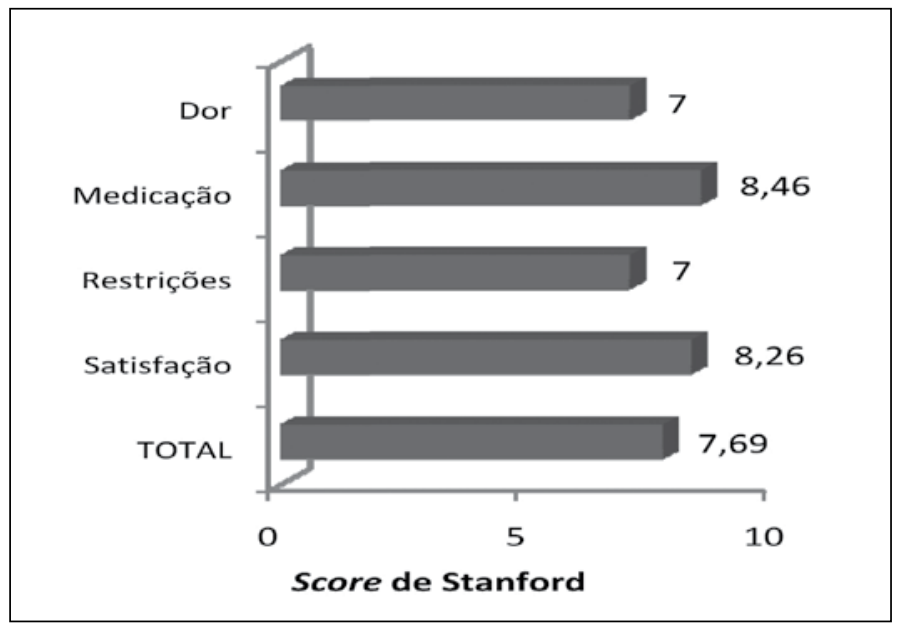

Figura 3B. Avaliação dos resultados.

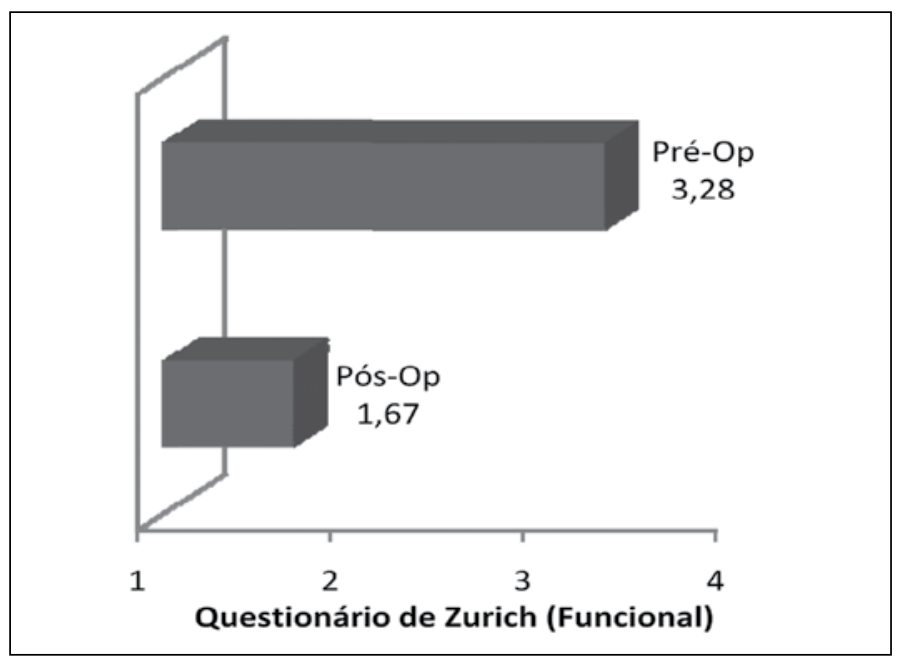

Figura 3C. Avaliação dos resultados.

Analisando o resultado funcional após tratamento (Tabela 2), observou-se uma relação significativa entre o Índice de Massa Corporal do doente e o Índice de Oswestry pós-operatório $(p=0,042$; $\mathrm{R}=+0,336)$. O tempo de baixa laboral pré-operatório foi o predictor mais forte do Índice de Oswestry pós-operatório ( $p<0,001$; 


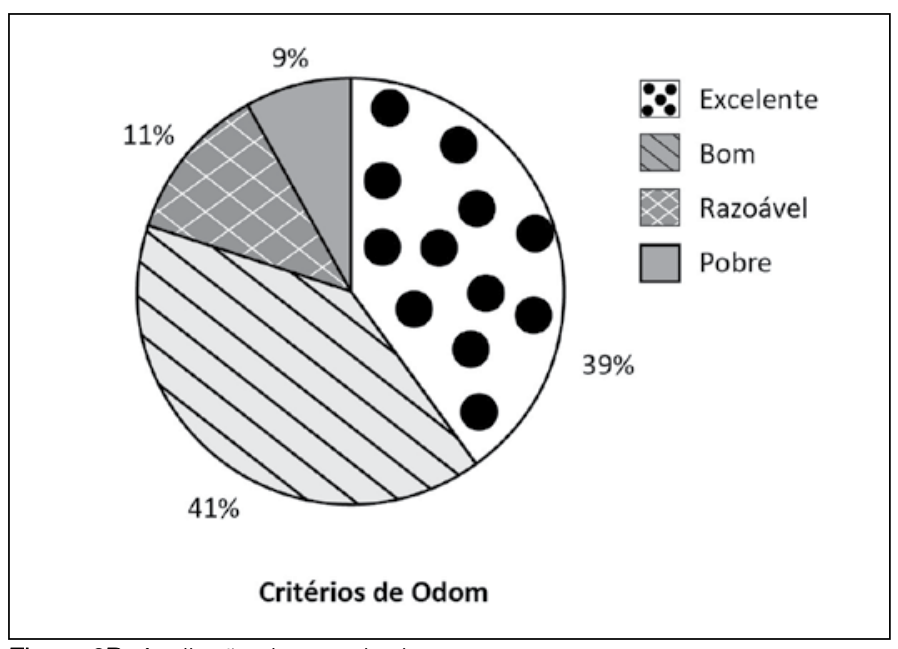

Figura 3D. Avaliação dos resultados.

Tabela 2. Determinantes do resultado funcional (Índice de Oswestry pós-operatório).

\begin{tabular}{|c|c|c|c|}
\hline \multicolumn{2}{|c|}{ Variável em Estudo } & $\mathbf{R}$ & $\mathbf{p}$ \\
\hline \multicolumn{2}{|c|}{ Índice de Massa Corporal ${ }_{\rho}$} & $+0,336$ & 0,042 \\
\hline \multicolumn{2}{|c|}{ Tempo de Baixa Laboral Pré-op $\rho$} & $+0,592$ & $<0,001$ \\
\hline \multicolumn{2}{|c|}{ Idade à Data da Cirurgia $\rho$} & $+0,342$ & 0,051 \\
\hline \multicolumn{2}{|c|}{ Tempo entre Cirurgias $\rho$} & $-0,254$ & 0,129 \\
\hline \multicolumn{2}{|c|}{ Tempo sem Sintomas entre cirurgias $\rho_{\rho}$} & $-0,220$ & 0,190 \\
\hline \multicolumn{2}{|c|}{ Tempo entre Sintomas e $2^{a}$ Cirurgia $\rho$} & 0,08 & 0,603 \\
\hline \multicolumn{2}{|c|}{ Variável em Estudo } & Valor Médio & $\mathbf{p}$ \\
\hline \multirow{2}{*}{$\mathrm{SexO}_{\mathrm{u}}$} & Masculino & $12,16 \%$ & \multirow{2}{*}{0,012} \\
\hline & Feminino & $26,33 \%$ & \\
\hline \multirow{2}{*}{$\begin{array}{l}\text { Necessidade de } \\
\text { Re-intervenção u }\end{array}$} & Sim & $28,33 \%$ & \multirow{2}{*}{0,271} \\
\hline & Não & $15 \%$ & \\
\hline
\end{tabular}

$\rho$ - Análise de Correlação de Spearman

u - Teste Mann-Whitney

$R=+0,592)$. Verificou-se uma diferença também entre sexos, com os doentes do sexo feminino a obter piores resultados (ODI pós-operatório médio de $26,33 \%$ vs $12,16 \%$ no caso dos homens; $p=0,012)$. Esta diferença não se correlacionou com a capacidade funcional pré-operatória (ODI médio de $66,17 \%$ no sexo feminino, e $61,52 \%$ nos pacientes do sexo masculino; $p=0,5$ ) nem se traduziu na avaliação subjectiva do resultado da cirurgia pelo paciente (grau "Satisfação" do Score de Stanford médio = 8,13 nas mulheres vs 8,32 no sexo masculino; $p=0,916$ ).

A idade à data da cirurgia, o tempo decorrido entre procedimentos, a necessidade de re-intervenção (terceira cirurgia), o tempo livre de sintomas e o intervalo de tempo entre a recorrência de sintomas e a cirurgia não se relacionaram de forma estatisticamente significativa com o Índice de Oswestry à data da reavaliação do paciente.

Na nossa série, a idade do paciente à data da cirurgia relacionou-se de forma estatisticamente significativa com a necessidade de terceiro procedimento cirúrgico (Tabela 3). Os doentes que necessitaram de ser operados uma terceira vez tinham em média
Tabela 3. Predictores da necessidade de terceira cirurgia.

\begin{tabular}{|c|c|c|c|c|}
\hline \multicolumn{2}{|c|}{ Variável em Estudo } & $\begin{array}{l}\text { Necessidade } \\
\text { de } 3^{\text {a }} \text { Cirurgia }\end{array}$ & $\begin{array}{l}\text { Sem } 3^{a} \\
\text { Cirurgia }\end{array}$ & $\mathbf{p}$ \\
\hline \multicolumn{2}{|c|}{ Idade à Data da Cirurgia (média) u } & 55 Anos & 45,4 Anos & 0,035 \\
\hline \multicolumn{2}{|c|}{ Índice de Massa Corporal (média) u } & $28,3 \mathrm{~kg} / \mathrm{m}^{2}$ & $26,8 \mathrm{~kg} / \mathrm{m}^{2}$ & 0,323 \\
\hline \multicolumn{2}{|c|}{$\begin{array}{l}\text { Tempo de Baixa Laboral Pré-op } \\
\text { (média) })_{u}\end{array}$} & 4,33 Meses & 3,25 Meses & 0,277 \\
\hline \multicolumn{2}{|c|}{ ODI Pré-operatório (média) u } & $67 \%$ & $62,2 \%$ & 0,606 \\
\hline \multicolumn{2}{|c|}{$\begin{array}{c}\text { Tempo entre } 1^{\text {a }} \text { e } 2^{\text {a }} \text { Cirurgias } \\
\text { (média) })_{u}\end{array}$} & 4,54 Anos & 4,86 Anos & 0,218 \\
\hline \multicolumn{2}{|c|}{$\begin{array}{l}\text { Tempo entre Sintomas e } 2^{\text {a }} \text { Cirurgia } \\
\text { (média) } \\
\text { u }\end{array}$} & 35,04 Meses & 9,7 Meses & 0,651 \\
\hline \multicolumn{2}{|c|}{ Variável em Estudo } & $\begin{array}{l}\text { Necessidade } \\
\text { de } 3^{\text {a }} \text { Cirurgia }\end{array}$ & $\begin{array}{l}\text { Sem } 3^{a} \\
\text { Cirurgia }\end{array}$ & p \\
\hline \multirow{2}{*}{$\operatorname{SexO}_{x 2}$} & Masculino & 9 & 26 & \multirow{2}{*}{0,161} \\
\hline & Feminino & 2 & 18 & \\
\hline
\end{tabular}

$x^{2}$ - Teste do Qui-quadrado

idade mais alta (55 anos vs 45,4 anos) à data da cirurgia de recidiva de hérnia discal.

O sexo do paciente, o Índice de Massa Corporal, o tempo de baixa laboral antes da cirurgia por recidiva, o nível de incapacidade funcional pré-operatório avaliado pelo Índice de Oswestry, o tempo decorrido entre primeira e segunda cirurgias e o tempo decorrido entre a recorrência de sintomas e a cirurgia não se relacionaram de forma estatisticamente significativa com a necessidade de terceira intervenção.

A necessidade de terceira cirurgia afirmou-se, na nossa série, como um factor de risco estatisticamente significativo para o não retorno ao trabalho prévio, assim como o Índice de Massa Corporal (Tabela 4). O tempo de baixa pré-operatório foi também um predictor com significância estatística do retorno laboral, sendo a mediana 1 mês de baixa pré-operatória nos doentes que retomaram o trabalho e 6,5 meses nos que não retomaram. Nenhum doente com baixa laboral prévia ao procedimento igual ou superior a 5 meses voltou ao trabalho.

O sexo e a idade do paciente, o Índice de Incapacidade de Oswestry pré-operatório e o intervalo de tempo entre a recorrência de sintomas e a cirurgia não se relacionaram de forma significativa com o regresso ao emprego.

\section{DISCUSSÃO}

As recidivas herniárias são reconhecidamente uma ocorrência em todas as séries cirúrgicas de hérnias discais lombares.

A taxa de recidiva a longo prazo após discectomia varia na literatura entre os $3,5 \%$ e os $12 \%^{6-12}$, reflectindo-se importantemente sobre a morbilidade e o custo dos cuidados de saúde envolvidos ${ }^{12}$, e parece depender de alguma forma do grupo etário envolvido?.

Mantêm-se hoje controversos vários aspectos relacionados com a doença, desde os critérios diagnósticos no estudo imagiológi$\mathrm{co}^{10,13-16}$ aos factores de risco para recidiva ${ }^{16}$. No tocante ao procedimento inicial, McGirt et a ${ }^{1{ }^{11}}$ associaram uma menor dimensão no defeito anular e uma maior agressividade na remoção discal a um menor risco de recidiva, apesar de este não se reflectir necessariamente de forma favorável em termos clínicos ${ }^{6}$ a longo prazo.

Na nossa série verificou-se uma variabilidade temporal importante na história natural da doença. Apesar de uma muito maior frequência das 
Tabela 4. Predictores do retorno laboral.

\begin{tabular}{|c|c|c|c|c|}
\hline \multicolumn{2}{|c|}{ Variável em Estudo } & $\begin{array}{l}\text { Retorno } \\
\text { Laboral }\end{array}$ & Sem Retorno & $\mathbf{p}$ \\
\hline \multicolumn{2}{|c|}{$\begin{array}{l}\text { Tempo de Baixa Laboral } \\
\text { Pré-op (mediana) u }\end{array}$} & 1 Mês & 6,5 Meses & 0,006 \\
\hline \multicolumn{2}{|c|}{$\begin{array}{l}\text { Índice de Massa } \\
\text { Corporal (média) u }\end{array}$} & $26,5 \mathrm{~kg} / \mathrm{m}^{2}$ & $31,8 \mathrm{~kg} / \mathrm{m}^{2}$ & 0,011 \\
\hline \multicolumn{2}{|c|}{ Idade à Data da Cirurgia (média) u } & 44,5 Anos & 46,5 Anos & 0,534 \\
\hline \multicolumn{2}{|c|}{ ODI Pré-operatório (média) u } & $62,1 \%$ & $61,5 \%$ & 0,740 \\
\hline \multicolumn{2}{|c|}{$\begin{array}{c}\text { Tempo entre Sintomas e } 2^{\mathrm{a}} \\
\text { Cirurgia (média) }_{u}\end{array}$} & 17,9 Meses & 11,8 Meses & 0,955 \\
\hline \multicolumn{2}{|c|}{ Variável em Estudo } & $\begin{array}{l}\text { Retorno } \\
\text { Laboral }\end{array}$ & Sem Retorno & $\mathbf{p}$ \\
\hline \multirow{2}{*}{$\begin{array}{l}\text { Necessidade de } \\
\text { Re-intervenção } \times 2\end{array}$} & Sim & 2 & 3 & \multirow{2}{*}{0,010} \\
\hline & Não & 21 & 4 & \\
\hline \multirow{2}{*}{$\operatorname{SexO}_{x 2}$} & Masculino & 18 & 4 & \multirow{2}{*}{0,964} \\
\hline & Feminino & 13 & 3 & \\
\hline
\end{tabular}

u - Teste Mann-Whitney

$x^{2}-$ Teste do Qui-quadrado

recidivas no $1^{\circ}$ ano após o procedimento inicial, estas ocorreram até ao $25^{\circ}$ ano.

A alta taxa de reprodutibilidade entre a sintomatologia antes da primeira e da segunda intervenções sugere uma associação entre eventos, favorecendo o conceito de recidiva.

O tempo médio entre cirurgias que encontrámos (58 meses) aproximou-se do de outras publicações ${ }^{17,18}$. O tempo médio desde a recorrência sintomatológica até ao tratamento cirúrgico (superior a 1 ano) pode significar, além do necessário espaço temporal para diagnóstico e tratamento conservador, algum grau de renitência da parte do clínico em partir para um novo tratamento cirúrgico.

De facto, as opções terapêuticas são várias, até no âmbito cirúrgico, não havendo também neste aspecto consenso acerca da mais adequada.

O papel que os tecidos de cicatrização e/ou fibrose resultantes de uma primeira cirurgia assumem no aumento da probabilidade de complicações per-operatórias ${ }^{19}$ favorece conceptualmente re-intervenções menos traumáticas. Tal reflecte-se nas propostas terapêuticas discutidas mais recentemente - nomeadamente a endoscópica ${ }^{20-25}$ que, quando comparadas à clássica abordagem microcirúrgica, se têm mostrado seguras ${ }^{24}$, estando descritos resultados sobreponíveis e por vezes até superiores em determinados parâmetros ${ }^{20,21,23}$. No outro extremo encontram-se as artrodeses, que apesar do maior grau de invasividade surgem também com bons resultados clínicos descritos ${ }^{26,27}$.

O procedimento padrão, na maioria dos centros e na ausência de outros achados que impliquem um procedimento adicional, mantém-se no entanto uma meticulosa ${ }^{28}$ revisão da microdiscectomia ${ }^{29}$, com elevadas taxas de eficácia (igualando ou ultrapassando, por vezes, os resultados da primeira cirurgia) ${ }^{18,30}$. Na amostra estudada, os resultados clínicos e funcionais subjectivos e objectivos, independentemente da escala aplicada, foram bastante positivos. Esta forma de tratamento cirúrgico mostrou-se ainda segura, com baixa incidência e gravidade de complicações. No entanto, um subgrupo considerável (um quinto) dos doentes precisou de uma terceira intervenção, que quando necessária ocorreu em média mais preco- cemente que a segunda (em menos de metade do tempo). Assim, não sendo este um estudo comparativo neste âmbito, ajuda a definir a revisão de discectomia como uma forma de terapêutica cirúrgica bem tolerada e com óptimos resultados, ainda que não definitivos numa proporção relevante de casos.

Várias revisões de segunda discectomia para tratamento de recidiva estão publicadas ${ }^{17,18,31,32}$. No contexto de resultados funcionais favoráveis e de elevadas taxas de retorno laboral, continua a manter-se alguma dificuldade em estabelecer factores pré-cirúrgicos que prevejam o outcome do paciente ${ }^{17,18}$.

Nesta série, a idade do paciente à data da cirurgia foi, em média, significativamente mais alta nos doentes que precisaram de terceiro procedimento, mas não foi um predictor significativo do resultado funcional (apesar da mesma tendência) ou do retorno laboral. O Índice de Massa Corporal do doente associou-se significativamente com o índice de incapacidade pós-operatória e com a probabilidade de não voltar a trabalhar, com desfavor dos doentes com maior massa.

O tempo de baixa laboral antes da cirurgia de recidiva herniária foi um predictor importante do resultado funcional do paciente e do seu retorno laboral.

O sexo do paciente associou-se de modo estatisticamente significativo com a capacidade funcional do paciente a longo prazo, em desfavor do sexo feminino, mas não encontrou correspondência na predicção da necessidade de terceira cirurgia e/ou do retorno laboral. Aliás, não parece haver diferença entre homens e mulheres na avaliação subjectiva que é feita do procedimento. Por outro lado, encontrámos nos doentes do sexo feminino um índice de massa corporal médio e um tempo de baixa laboral mediano mais altos, não sendo possível na análise da amostra determinar se estas variáveis se comportam como independentes.

$\mathrm{Na}$ revisão de literatura realizada não encontrámos estas associações entre dados pré-operatórios e resultados funcional e laboral a longo prazo.

\section{LIMITAÇÕES}

Tratando-se de um estudo retrospectivo, não nos foi possível recolher dados suficientes sobre outras variáveis que poderiam influenciar os resultados, como a imagiologia pré e pós-operatória (redução da altura discal, protrusão versus extrusão discal, papel da fibrose peri-radicular), duração e tipo de tratamento conservador pré-operatório, técnica cirúrgica (sequestrectomia versus discectomia "agressiva") ou a reabilitação funcional pós-operatória. Atendendo ao número de cirurgias realizadas anualmente, a análise destas correlações pode beneficiar com uma amostra de maiores dimensões e com dados colhidos de forma prospectiva.

\section{CONCLUSÕES}

O intervalo de tempo entre a cirurgia e a ocorrência de uma recidiva de hérnia discal lombar é extremamente variável. A sintomatologia aquando da recidiva é muito sobreponível à do primeiro evento. $\mathrm{O}$ tratamento cirúrgico desta entidade possibilita muito bons resultados em termos sintomáticos e funcionais, independentemente do teste aplicado, e com influência no retorno à actividade profissional. Um subgrupo de pacientes, cujos sintomas se perpetuam condicionando diferentes graus de incapacidade, pode ser em parte previsto com base em variáveis clínicas e epidemiológicas. O presente trabalho pretende contribuir para o reconhecimento dessas mesmas características, realçando alguns factores que se correlacionam mais fortemente com o resultado e que deverão ser considerados quando é proposto o tratamento. 


\section{REFERÊNCIAS}

1. Million $\mathrm{R}$, Hall W, Nilsen $\mathrm{KH}$, Baker RD, Jayson Ml. Assessment of the progress of the back-pain patient 1981 Volvo Award in Clinical Science. Spine (Phila Pa 1976). 1982;7(3):204-12.

2. Fairbank JC, Pynsent PB. The Oswestry Disability Index. Spine (Phila Pa 1976). 2000;25(22):2940-52.

3. Stucki G, Daltroy L, Liang MH, Lipson SJ, Fossel AH, Katz JN. Measurement properties of a self-administered outcome measure in lumbar spinal stenosis. Spine (Phila Pa 1976). 1996;21(7):796-803.

4. Carragee EJ, Kim DH. A prospective analysis of magnetic resonance imaging findings in patients with sciatica and lumbar disc herniation. Correlation of outcomes with disc fragment and canal morphology. Spine (Phila Pa 1976). 1997;22(14):1650-60.

5. Odom GL, Finney W, Woodhall B. Cervical disk lesions. J Am Med Assoc. 1958;166(1):23-8.

6. McGirt MJ, Ambrossi GL, Datoo G, Sciubba DM, Witham TF, Wolinsky JP, et al. Recurrent disc herniation and long-term back pain after primary lumbar discectomy: review of outcomes reported for limited versus aggressive disc removal. Neurosurgery. 2009;64(2):338-44

7. Kim MS, Park KW, Hwang C, Lee YK, Koo KH, Chang BS, et al. Recurrence rate of lumbar disc herniation after open discectomy in active young men. Spine (Phila Pa 1976). 2009;34(1):24-9

8. Hakkinen A, Kiviranta I, Neva MH, Kautiainen H, Ylinen J. Reoperations after first lumbar disc herniation surgery; a special interest on residives during a 5 -year follow-up. BMC Musculoskelet Disord. 2007;8:2.

9. Gaston P, Marshall RW. Survival analysis is a better estimate of recurrent disc herniation. J Bone Joint Surg Br. 2003;85(4):535-7.

10. Erbayraktar S, Acar F, Tekinsoy B, Acar U, Guner EM. Outcome analysis of reoperations after lumbar discectomies: a report of 22 patients. Kobe J Med Sci. 2002;48(1-2):33-41.

11. McGirt MJ, Eustacchio S, Varga P, Vilendecic M, Trummer M, Gorensek M, et al. A prospective cohort study of close interval computed tomography and magnetic resonance imaging after primary lumbar discectomy: factors associated with recurrent disc herniation and disc height loss. Spine (Phila Pa 1976). 2009;34(19):2044-51.

12. Ambrossi GL, McGirt MJ, Sciubba DM, Witham TF, Wolinsky JP, Gokaslan ZL, et al. Recurrent lumbar disc herniation after single-level lumbar discectomy: incidence and health care cost analysis. Neurosurgery. 2009;65(3):574-8.

13. Bialecki J, Lukawski S, Milecki M, Lachowicz W. Differential diagnosis of post-surgery scars and recurrent lumbar disc herniation in MRI. Ortop Traumatol Rehabil. 2004;6(2):172-6.

14. Mullin WJ, Heithoff KB, Gilbert TJ Jr, Renfrew DL. Magnetic resonance evaluation of recurrent disc herniation: is gadolinium necessary? Spine (Phila Pa 1976). 2000;25(12):1493-9

15. Akeson $P$, Annertz M, Kristoffersen DT, Jonsson E, Holtas S. MR imaging in recurrent pain after back surgery. A comparative study using standard and high doses of gadolinium contrast agents. Acta Radiol. 1996;37(6):858-62.
16. Kim KT, Park SW, Kim YB. Disc height and segmental motion as risk factors for recurrent lumbar disc herniation. Spine (Phila Pa 1976). 2009;34(24):2674-8.

17. Dai $L Y$, Zhou $Q$, Yao WF, Shen $L$. Recurrent lumbar disc herniation after discectomy: outcome of repeat discectomy. Surg Neurol. 2005;64(3):226-31.

18. Suk KS, Lee HM, Moon SH, Kim NH. Recurrent lumbar disc herniation: results of operative management. Spine (Phila Pa 1976). 2001;26(6):672-6.

19. Kayaoglu CR, Calikoglu $C$, Binler $S$. Re-operation after lumbar disc surgery: results in 85 cases. J Int Med Res. 2003;31(4):318-23.

20. Ruetten S, Komp M, Merk H, Godolias G. Recurrent lumbar disc herniation after conventional discectomy: a prospective, randomized study comparing full-endoscopic interlaminar and transforaminal versus microsurgical revision. J Spinal Disord Tech. 2009;22(2):122-9.

21. Zhou Y, Wang J, Chu TW, Li CQ, Zhang ZF, Wang WD, et al. [Micro-endoscopic strategies and options for recurrent lumbar disc herniation]. Zhonghua Wai Ke Za Zhi. 2008:46(19):1475-9.

22. Hoogland T, van den Brekel-Dijkstra K, Schubert M, Miklitz B. Endoscopic transforaminal discectomy for recurrent lumbar disc herniation: a prospective, cohort evaluation of 262 consecutive cases. Spine (Phila Pa 1976). 2008;33(9):973-8.

23. Le H, Sandhu FA, Fessler RG. Clinical outcomes after minimal-access surgery for recurrent lumbar disc herniation. Neurosurg Focus. 2003;15(3):E12.

24. Isaacs RE, Podichetty $V$, Fessler RG. Microendoscopic discectomy for recurrent disc herniations. Neurosurg Focus. 2003;15(3):E11.

25. Ahn Y, Lee SH, Park WM, Lee HY, Shin SW, Kang HY. Percutaneous endoscopic lumbar discectomy for recurrent disc herniation: surgical technique, outcome, and prognostic factors of 43 consecutive cases. Spine (Phila Pa 1976). 2004;29(16):E326-32.

26. Chen Z, Zhao J, Liu A, Yuan J, Li Z. Surgical treatment of recurrent lumbar disc herniation by transforaminal lumbar interbody fusion. Int Orthop. 2009;33(1):197-201.

27. FuTS, Lai PL, Tsai TT, Niu CC, Chen LH, Chen WJ. Long-term results of disc excision for recurrent lumbar disc herniation with or without posterolateral fusion. Spine (Phila Pa 1976). 2005;30(24):2830-4.

28. Stambough JL. Recurrent same-level, ipsilateral lumbar disc herniation. Orthop Rev. 1994;23(10):810-6

29. Swartz KR, Trost GR. Recurrent lumbar disc herniation. Neurosurg Focus. 2003;15(3):E10.

30. Papadopoulos EC, Girardi FP, Sandhu HS, Sama AA, Parvataneni HK, O'Leary PF, et al. Outcome of revision discectomies following recurrent lumbar disc herniation. Spine (Phila Pa 1976). 2006;31(13):1473-6.

31. Kloc W, Imielinski BL, Wasilewski W, Jende P, Puzyrewski R. [Repeated operations for recurrent lumbar disc herniations]. Neurol Neurochir Pol. 1999;33(6):1367-72.

32. Herron L. Recurrent lumbar disc herniation: results of repeat laminectomy and discectomy. J Spinal Disord. 1994;7(2):161-6. 